\title{
Responsabilidad e inclusión social: Evaluación de Atributos personales
}

\author{
Responsibility and social inclusion: Personal attributes assessments
}

\author{
Francisco Manuel Morales Rodríguez*, Manuel Alejandro Narváez Peláez* Juan Ramón Pereira Docampo** \\ *Universidad de Málaga, **Asociación por la divulgación, investigación y docencia de la psicología
}

\begin{abstract}
Resumen
Se presenta una evaluación de atributos personales que serán de utilidad para analizar su relación con actitudes y comportamientos socialmente responsables. Los participantes han sido 36 universitarios de la titulación de Logopedia de la Facultad de Psicología con edades comprendidas entre los 18 y los 47 años de la provincia de Málaga que pasaron un cuestionario para la evaluación del rol de género. Se encuentran relaciones entre las variables atributos personales y comportamientos socialmente responsables (a mayor puntuación en el atributo 1 (Muy agresivo), en menor medida se participa en campañas de cuidado del medio ambiente, etc.

Palabras clave: educación inclusiva, rol, género, responsabilidad social
\end{abstract}

\begin{abstract}
Personal attributes assessments have been carried out so as to analyse socially responsible attitudes and behaviours. 36 students of Logopaedics at psychology faculty from

University of Málaga, aged 18 to 47, undertook a survey on personal attributes assessment (gender role). Connections between personal attributes and socially responsible behaviours are found. The higher the grades in attribute 1 (Very aggressive); the less the participation in Environmental Protection campaigns.

Keywords: inclusive education, gender, role, social responsibility
\end{abstract}

El presente trabajo se enmarca en el Proyecto de Innovación Educativa (PIE13-0031; PIE10-127), en el que para formar ciudadanos y ciudadanas europeos se fomentan competencias relacionadas con la responsabilidad social y educación inclusiva. Una de las variables relevantes para considerar en este ámbito es el rol de género.

En la actualidad cada vez se pueden localizar más estudios cuya línea temática se centran directamente o consideran entre sus variables la importancia del rol de género (Jiménez-García et al., 2013; Laliena, 2010; Martínez-Leon, Arcas-Lario, y García-Hernandez, 2011).

Con respecto al género, estudios previos (Plazas et al., 2010; Redondo y Guevara, 2012) encuentran mayor comportamiento prosocial y menos conducta agresiva en el género femenino en comparación con el masculino. Otros estudios encuentran también en la mujer mayor predisposición empática (Galán, Romero, Morillo y Alarcón, 2014; Mestre-Escrivá, Frías-Navarro, y Samper-García, 2004).
Precisamente en este trabajo se realiza una evaluación de atributos personales que serán de utilidad para analizar su relación con actitudes y comportamientos socialmente responsables.

\section{Método}

\section{Participantes}

Los participantes han sido 36 universitarios de la titulación de Logopedia de la Facultad de Psicología con edades comprendidas entre los 18 y los 47 años de la provincia de Málaga que pasaron un cuestionario para la evaluación de atributos personales (rol de género).

\section{Instrumentos o Materiales}

En el presente trabajo se han aplicado estos instrumentos:

1) Cuestionario de autoatribución de comportamientos socialmente responsables en universitarios (CACSR, Davidovich, Espina, Navarro y Salazar, 2005). Este instrumento incluye dos escalas: una de frecuencia y otra de intencionalidad. Está compuesto por un total de 40 ítems según escala tipo Likert de cinco escalones según se trate de la escala de frecuencia $(1=$ Nunca; $2=$ Casi nunca; $3=$ A veces; $4=$ Casi siempre y $5=$ Siempre) o la escala de intención ( $1=$ No aplica; 2 = Beneficio personal; $3=$ No tengo clara la intención; $4=$ Beneficio para otros y $5=$ Beneficio mutuo). Ambas escalas están formadas por las siguientes diez categorías: Responsabilidad laboral/académica, actividades de voluntariado, ayuda social, actividades religiosas, convivencia social, responsabilidad cívica, autocuidado, desarrollo cultural, ecología y medio ambiente, y respeto por espacios compartidos.

2) También se ha aplicado el cuestionario ya consolidado en numerosas aplicaciones denominado Cuestionario de Atributos Personales (PAQ, Spence y Helmreich, 1978). Las respuestas de este cuestionario son tipo Likert de 5 opciones: A, B, C, D, E.

\section{Procedimiento}

Los datos se aplicaron en el grupo de clase y fueron de carácter voluntario asegurando tanto la confidencialidad de la información como el hecho de que no se comparará por grupos $\mathrm{y}$ tendrán un tratamiento global sin particularizar ni comparar entre personas o grupos. Dichos cuestionarios además se relacionaron con la línea temática para impartir en la asignatura. 


\section{Resultados}

Los resultados demuestran que en esta muestra las puntuaciones medias más elevadas se han obtenido en los ítems que forman parte de la sub-escala Feminidad $(3.86 ; 4.11 ; 4.14$ y 3.94 respectivamente en los ítems Muy emocional; Muy amable; Muy comprensivo/a con los demás; Muy cálido/a en las relaciones con los demás según escala Likert de cinco puntos). En la sub-escala de Masculinidad la puntuación media más elevada se ha obtenido en el ítem Muy independiente (4.00).

Se encuentran relaciones entre las variables atributos personales y comportamientos socialmente responsables (por ejemplo, a mayor puntuación en el atributo 1 (Muy agresivo), que forma parte de las subescalas Masculinidad-Feminidad en menor medida se participa en campañas de cuidado del medio ambiente; menos interés por informarse en los acontecimientos nacionales e internacionales y menos afirman realizar donaciones de dinero a campañas de ayuda social.

A mayor puntuación en el atributo 9 (Muy servicial con los demás) que forma parte de ambas subescalas Masculinidad-Feminidad en mayor medida manifiestan participar en la organización de campañas de ayuda solidaria para la entrega de alimentos, ropas, etc).

A mayor puntuación en el atributo 16 (Puedes tomar decisiones fácilmente), que forma parte de la Escala de Masculinidad según sus autores, mayor puntuación se obtiene en la autoatribución del comportamiento socialmente responsable referido a mantener en los espacios públicos una actitud de cuidarlos.

Puede destacarse también que las puntuaciones medias superiores se han obtenido en los comportamientos socialmente responsables relacionados con el bien común y la equidad social ("pienso que todos tenemos derecho a las mismas cosas"); la integridad; la aceptación y aprecio de la diversidad ("acepto a los demás independientemente de su orientación sexual, sexo, raza, condición física", etc) y el respeto a espacios reservados para ancianos, etc.

A continuación se muestran en la Tabla 1 los resultados estadísticamente significativos más relevantes. Solo se muestran aquellos resultados estadísticamente significativos.

Tabla 1.

Correlaciones de Pearson entre atributos y comportamientos sociales

\begin{tabular}{lllll}
\hline Atributo & & & & \\
\hline & $\mathrm{C} 11$ & $\mathrm{C} 12$ & $\mathrm{C} 13$ & $\mathrm{C} 14$ \\
Atributo 1 & -.21 & -.31 & -.23 & $-.42^{* *}$ \\
Atributo 9 & -.15 & -.03 & $.37 *$ & .20 \\
Atributo 16 & $.34 *$ & -.12 & .01 & -.02 \\
\hline
\end{tabular}

${ }^{* *} p<.01 ;{ }^{*} p<.05 ; \mathrm{C} 11=$ Mantengo en los espacios públicos una actitud de cuidarlos; $\mathrm{C} 12=$ Cumplo responsablemente tareas, trabajos y evaluaciones: $\mathrm{C} 13=$ Organizo campañas de ayuda solidaria; $\mathrm{C} 14$ = Dono dinero a campañas de ayuda social.

\section{Conclusiones}

Se concluye señalando la utilidad de la información que proporcionan estos datos considerando la importancia de la variable rol de género y sus relaciones con otras variables para una educación inclusiva, prevención de la violencia de género y contribuir a la mejora de la convivencia social.

Los resultados apuntan que se pueden establecer relaciones aunque no sean desde un punto de vista causal entre los atributos personales con los que se identifica el alumnado y la percepción y la atribución de comportamientos socialmente responsables evaluados a través del CACSR. De forma más concreta, se ha obtenido que, en esta muestra de universitarios, la puntuación más elevada se muestra en la autoatribución de comportamientos socialmente responsables relacionados con aspectos como la importancia de la equidad y de la atención a la diversidad.

Aunque los resultados de este estudio no son concluyentes en ese sentido, en otros trabajos (Álvarez-Torres, 2013) se encuentra en el género femenino una mayor capacidad de aserción en comparación con el masculino.

Es necesario ampliar el tamaño de la muestra de este trabajo para profundizar en el papel que puede jugar el rol de género (atributos personales) en la autoatribución de comportamiento $y$ actitudes en universitarios. Asimismo se pueden estudiar estos aspectos en estudiantes de otras titulaciones y centros.

\section{Referencias}

Álvarez-Torres (2013). Cómo influye la autoestima en las relaciones interpersonales. Universidad de Almería, Almería, España. Trabajo recuperado de:

http://repositorio.ual.es:8080/jspui/bitstream/10 835/2298/1/Trabajo.pdf

. Davidovich, M. P., Espina, A., Navarro, G. y Salazar, L. (2005). Construcción y estudio piloto de un cuestionario para evaluar comportamiento socialmente responsables en estudiantes universitarios. Revista de Psicología de la Universidad de Chile, 14(1),125-139.

. Galán, J. M., Romero, M., Morillo, R. y Alarcón, J. M. (2014). Descenso de empatía en estudiantes de enfermería y análisis de posibles factores implicados. Psicología Educativa, 20, 53-60. doi: 10.1016/j.pse.2014.05.007

Jiménez, R., Luque-Budia, A., Delgado-Ríos, P., Rojo-Villalba, M., Ruiz-Veguilla, M., Aguado-Romeo, M. J., ... Moya-Morales, M. (2013, Junio). Evaluación del sexismo ambivalente en las y los profesionales de la red de salud mental de Andalucía. Proceedings of 6th International and 11th National Congress of Clinical Psychology, Santiago de Compostela, España. 
Laliena, M. C. (2010). Perspectiva de gé ero y enfoque de responsabilidad social en el estudio del sector textil: aportaciones a la cooperación al desarrollo. Zaragoza: Prensas Universitarias de Zaragoza.

Plazas, E. A., Morón, M. L., Santiago, A., Sarmiento, H., Ariza, S. E. y Patiño, C. D. (2010). Relaciones entre iguales, conducta prosocial y género desde la educación primaria hasta la universitaria en Colombia. Universitas Psychologica, 9(2), 357-369.

Mestre-Escrivá, V., Frías-Navarro, M., y Samper-García, P. (2004). La medida de la empatía: Análisis del interpersonal reactivity index. Psicothema, 16(2), 255-260.

Redondo, J. y Guevara, E. (2012). Diferencias de género en la prevalencia de la conducta prosocial y agresiva en adolescentes de dos colegios de la ciudad de Pasto. Revista Virtual Universidad Católica del Norte, 36, 173-192.

Martínez-Leon, I. M., Arcas-Lario, N., \& García-Hernandez, M. (2011). La influencia del género sobre la responsabilidad social empresarial en las entidades de economía social. (the influence of gender on corporate social responsibility in social economy enterprises. with english summary.). Revista De Estudios Cooperativos, 105, 143-172. Recuperado de http://0-search.proquest.com.jabega.uma.es/doc view/919222084? accountid=14568

Spence, J. T. \& Helmreich, R. L. (1978). Masculinity and femininity: Their psychological dimensions, correlates and antecedents. Austin: University of Texas Press. 